Article

\title{
State of the art neurosurgical OP Theater Necesity, not option!
}

Răzvan Onciul, Andrei Marinescu, Georgeta Popa, A.V. Ciurea 


\title{
State of the art neurosurgical OP Theater Necesity, not option!
}

\author{
Răzvan Onciul1', Andrei Marinescu' ${ }^{1}$, Georgeta Popa ${ }^{2}$, A.V. Ciurea ${ }^{3,4}$ \\ ${ }^{1}$ Student, "Carol Davila” UMPh, Bucharest, ROMANIA \\ ${ }^{2}$ Neuroradiology Department, Sanador Hospital, Bucharest, ROMANIA \\ ${ }^{3}$ Neurosurgery Department, Sanador Hospital, Bucharest, ROMANIA \\ ${ }^{4}$ Neurosurgery Department “Carol Davila” UMPh, Bucharest, ROMANIA
}

\begin{abstract}
Cerebral activity is extremely complex and requires a super diagnosis investigation - there is no limit to this super investigation, especially in the case of expansive tumor or non-tumoral le-sions. Modernization of the diagnosis in these lesions has gained Science Fiction aspects. Adapting to those changes, operatory blocks and neurosurgeons all around the world have been improved to the same extent in order to obtain the most perfect results with keeping the quality of life. The modern slogan of neurosurgery is "MAXIMAL SAFE RESACTION". Continu-ing on this theme we can state that the equipment of the surgical apparatus is very special and extremely expensive. Complex but in accordance with one of the most exquisite agglomeration of atoms in the entire known Universe, the human brain!
\end{abstract}

Key words: neurosurgery, MRI, OP theather, neuronavigation, operating microscop, hibryd OP room, robotic surgery, future, science-fiction

\section{Introduction}

Inicidence of intracranial or intrarahidian lesion processes have lately increased. On one hand, more and more complex investigative means have appeared, and on the other hand, the number of primary and secondary neurosurgical disease in the whole European pathology has increased.
In these conditions, the smallest neurology, ophtalmologic and ENT signs should be considered taking a proper clinical examination and carrying out paraclinical investigations: MRI with all facilities-native, contrast, angio and FLAIR, STIR, DTI etc. 


\section{Material}

After complete diagnosis of expansive lesion in the nervous system we must intervene quickly because there is no place for expansion in the intracranial space and the intracahidian space. In fact, the current name in all such situations is intracranial expansive (PEIC) or intrara-hidian process. Neurosurgical intervention should be done in a timely manner with all possible facilities to achieve a good result with restitutio ad integrum.

A modern neurosurgical room needs a proper operating table for special operator procedures - Maquet - plus the right external illuminators. The patient's head must be secured in the Mayfield device to prevent any movement during craniotomy maneuvers and laso to allow intracranial injuries to be tackled in the most dificult locations. (Figure 1)

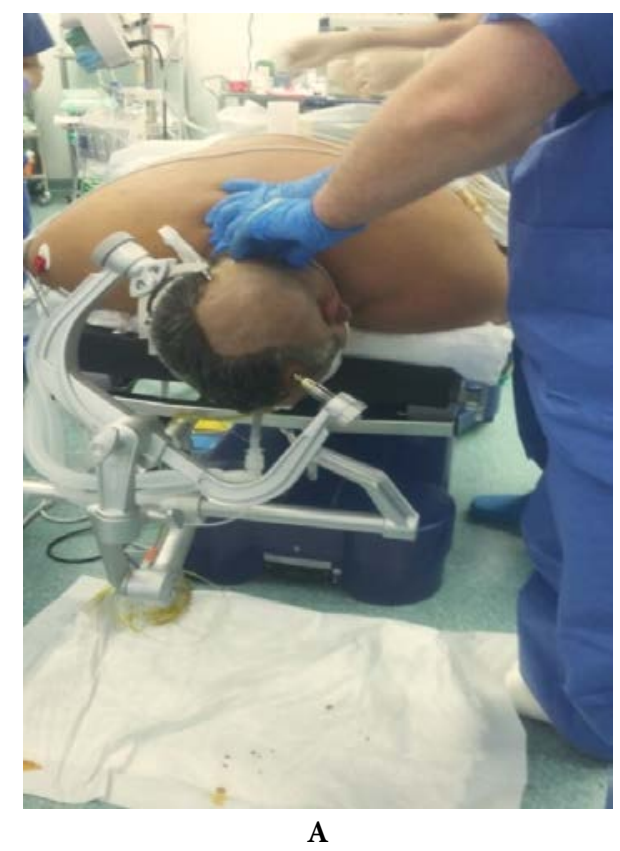

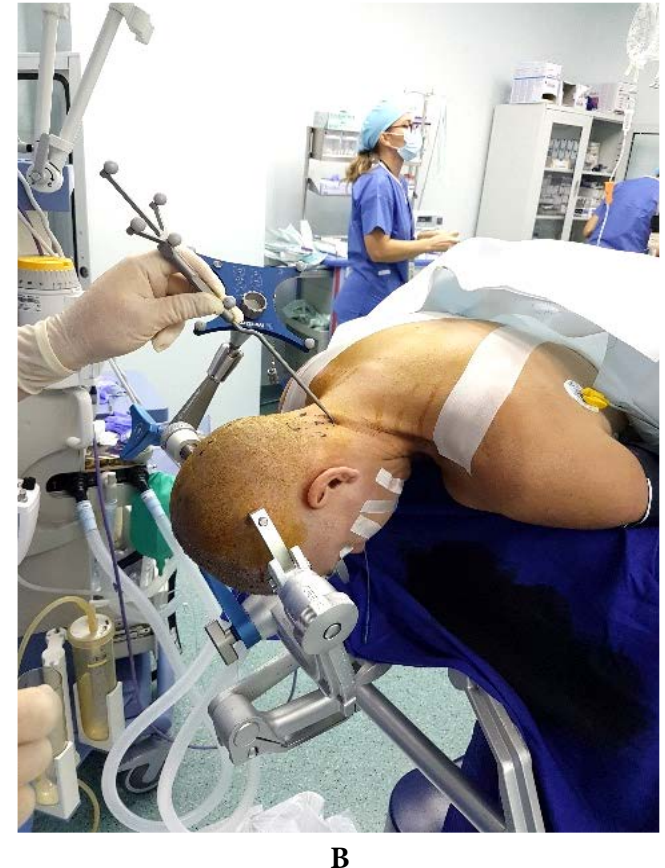

Figure 1 - Marker for neuronavigation and Mayfield fixator $(A, B)$

Neuronavigation is used for accurate lesion delineation and targeted opening based on neuronavigation data from previous MRI and CT-scan. Of particular importance are the neuroanesthesia conditions that have to be perfect: hypnosis, sedation, analgesic medication, brain relaxation through depletion treatment.

Intraoperative and postoperative MRI are a routine in most medical centers of civilized countries.

Neurosurgical approach is done minimally invasive under the operator microscope with avoidance of elocvent areas, which is done by obtaining data from functionally MRI correlated with neuronavigation. The technology behind the operating microscopes 
has improved a lot, reaching major performances (ex: Pentero 900). Currently in Bucharest there are 7 Pentero Microscopes with an individual value of 260.000 euros (Fig. 2). They bring a major benefit to both the surgeon and the patient: the operations are made easier, more elegant and comfortable for the neurosurgical team and with fewer risks for the patient due to the visualization of both the lesion and the surrounding vasculopathy structures.

The exerezis of the expansive process is extremely slow, careful, with the exact delimitation of the lesional entity to normal brain tissue. The exeretion must be made as complete as possible, but with the preservation of the quality of life. Hemostasis is performed very carefully in order not to leave intracerebral hemorrhagic areas, then try to restore CSF pathways, open the arachnoid spaces and basal cisterns. (Figure 3)

The cerebral substance is continuously irrigated with "warm" (at body temperature) saline solution. After that, the total ablation of the lesion is made, with the application of hemostatic substances on the surface - based on human fibrins autologous (AFS) - thereby stopping any kind of diffuse bleeding. Microneurosurgical instrumentation must be of the highest quality, of course titanium, perfectly adapted to neurosurgical procedures that require a finesse imposed by the complex and sophisticated brain structures. (Figure 4)

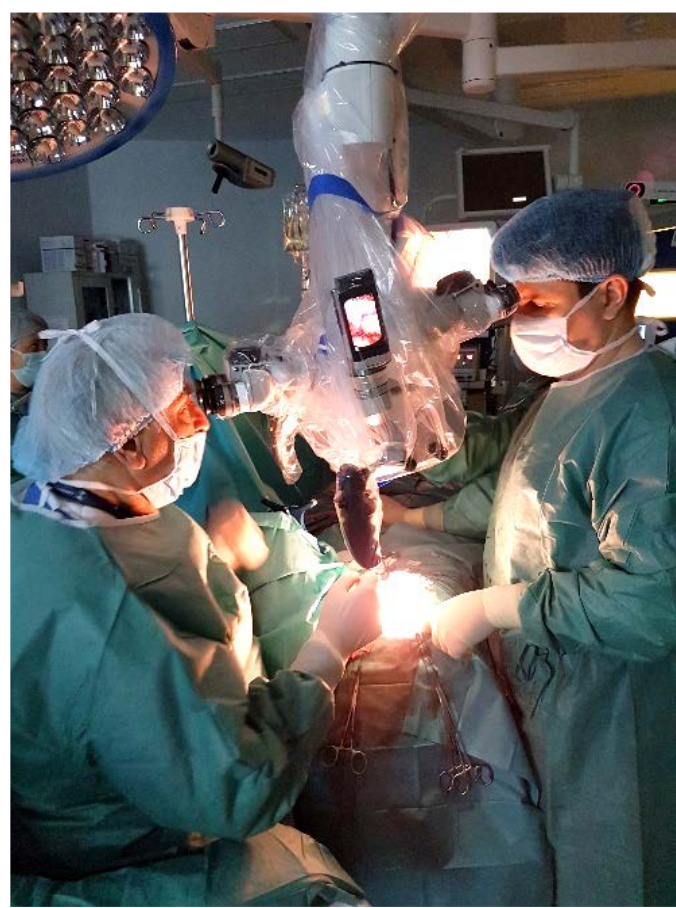

A

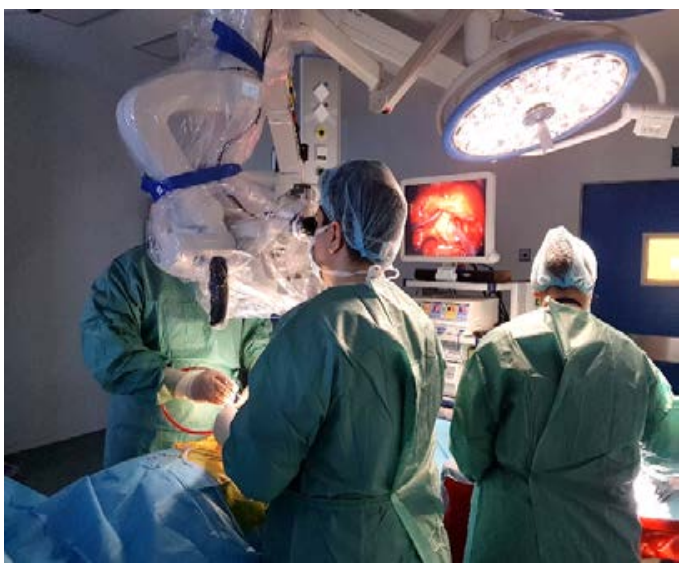

B

Figure 2 - Operating theatre (Pentero 900 microscope) (A, B) 


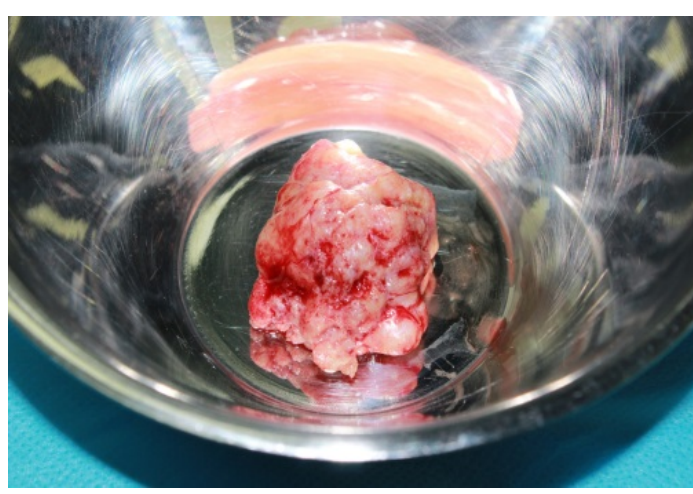

A

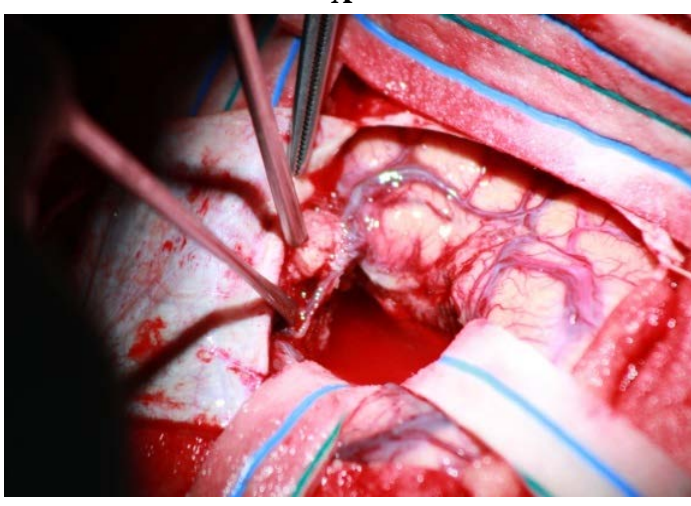

B

Figure 3 - Tumor mass (meningioma) completely removed

It must be used Watertight closer of dura mater or without dural plastia or artificial dura, again applying the AFS product for stoping the rest of CSF.

Reimplantation and perfect fixation of the bone calvaria it is an impotant element for cerebral neural volumetry and physiology. Closure in perfect anatomical planes is necessary to restore the adjacent structures.

All of these surgical procedures other than patient benefit recognized in all publications are also an element of neurosurgical training. (1)

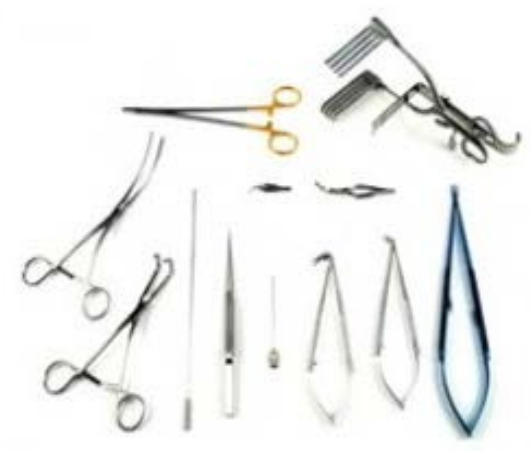

A

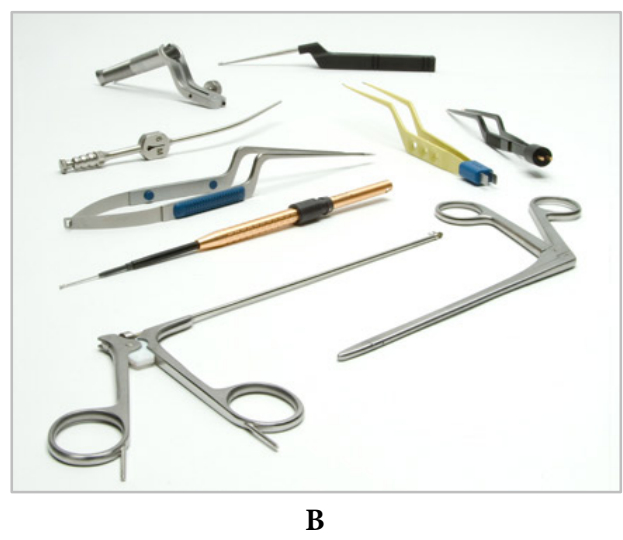

Figure 4 - Microsurgical instruments (A, B)

Ideally, every surgical operation in the operating theater must be video recorded to be reviewed later, from a forensic point of view, but especially used as material in the education process, helping to speed up the learning curve.

Regarding vascular pathology this involves for the future an interdisciplinary angio suit in which the angiographic diagnosis is completed with the open microsurgical approach (Clipping) and also with the current endovascular procedures (Coils). (2)

Always present in the modern OR must be: the special neurosurgery kits for craniocerebral or spinal interventions, cranial nerve 
monitoring devices, hydrocephalic drainage kit, lumbar CSF drainage kit, etc.

Neuroendoscopy is also a very important point to achieve in minimally invasive intraventricular interventions. This is necessary to restore CSF circulation and to resect injuries at the cerebral ventricular structures level. (3)
Curently the biggest improvement of a neurosurgical theather is the hybrid operating room in witch $\mathrm{u}$ can add to the standard $\mathrm{OR}$ other devices such as: surgical angiography, CArms, intraoperative/postoperative CT scan or even intraoperative/postoperative MRI. (Figure 5)

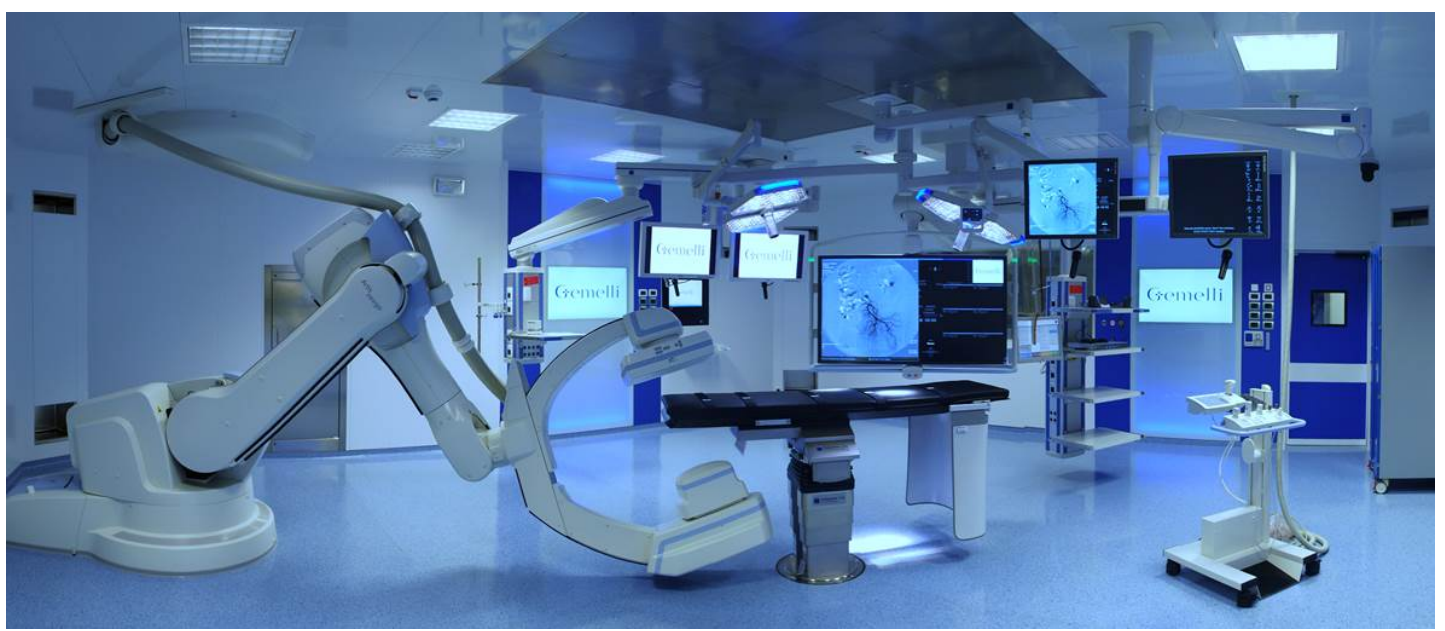

Figure 5 - Hybrid operating room

A hybrid operating room is a major investment; only the purchase cost is between 1 and 2 million euros; added to these are the high costs of remodeling and maintenance. However, the economic benefit is reflected in the increasing demand for complicated surgeries. The surgical act become less time consuming with shorter periods of hospitalization for patients due to more accessible methods of intervention. In addition, intraoperative imaging also facilitates immediate quality assurance that leads to a reduction in errors.

\section{Perspectives}

The future is the robotic surgery which is being attempted to be implemented in the field of functional neurosurgery (involuntary movements) $(4,5,6)$. Robotic systems are not widely used in neurosurgery compared to other areas of medicine such as urology, cardiology and gastroenterology. This is due to the anatomical challenges of such a complex and space-limited organ and to the fact that the brain includes extremely sensitive tissue. (Figure 6) (7). 


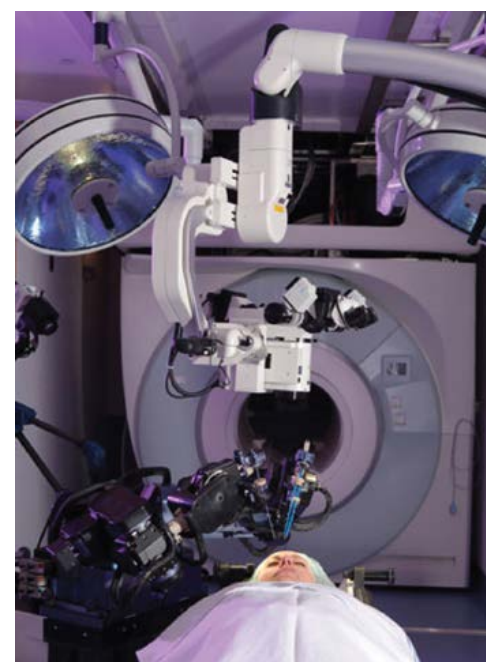

Figure 6 - NeuroArm (Univestity of Calgary, Alberta, Canada)

All these very costly elements are necessary for a better outcome and restitution of the patient's quality of life. At least, in neurosurgery science-fiction technology in the operating theater is not an option, it is a necessity!

\section{Conclusion}

It is impossible to predict the future evolution of neuroimaging diagnosis in cerebral lesions, in particular. Technological developments compete for the development of this complex chapter. Accordingly, the entire operator palette with all the devices included is constantly adapting to operating results as perfect as possible with patient integration into family, professional and, of course, social work.

The evolution of the entire technology in $\mathrm{OP}$ theather is extremely fast and adapted to the latest acquisitions of neuroanatomy, neurophysiology, neuroscience and especially neuroimaging.

\author{
Abbreviations \\ $\mathrm{CSF}=$ Cerebro spinal fluid \\ $M R I=$ Magnetic Resonance Imaging \\ CT= Computed Tomography \\ $S F=$ Science-fiction \\ $\mathrm{OR}=$ Operating room \\ AFS $=$ Autologous fibrin solution \\ FLAIR=Fluid Attenuation Inversion Recovery \\ $S T I R=$ Short $T 1$ Inversion Recovery \\ $D T I=$ Diffusion tensor imaging
}

\section{References}

1.Dammann P. Breyer T. Wrede K. H., Stein K. P., Wanke I., Grams E., Gizewski R. E., Schlamann M., Forsting M., Sandalcioglu E.. "Treatment of Complex Neurovascular Lesions: An Interdisciplinary Angio Suite Approach.” Therapeutic Advances in Neurological Disorders 7.1 (2014): 60-70.

2.Konakondla S., Reginald F, and Schirmer C.M.. "Simulation Training in Neurosurgery: Advances in Education and Practice." Advances in Medical Education and Practice 8 (2017): 465-473.

3.Rohde V. Behm T., Ludwig H. Wachter D. "The Role of Neuronavigation in Intracranial Endoscopic Procedures." Neurosurgical Review 35.3(2018): 351-358. 4.Madhavan K.,Kolcun J.P.G., Chieng L. O.,Wang M. Y., "Augmented-reality integrated robotics in neurosurgery: Are we there yet?," Neurosurgical Focus (2017), 42, no. 5, 5.De Benedictis A., Palma P.,Amante P., Rizzi M., Marras C. F., Trezza A., Carai A., Genovese E., Procaccini E., Messina R., Randi F., Cossu S., Esposito G., "Robotassisted procedures in pediatric neurosurgery," Neurosurgical Focus (2017), 42, no. 5,

6.Benabid AL, Hoffman D, Seigneuret E, Chabardes S. Robotics in neurosurgery: which tools for what? Acta Neurochir Suppl. 2006;98:43-50.

7.Amirabdollahian F., Livatino S.,Vahedi B., Gudipati R., Sheen P., Gawrie-Mohan S., and Vasdev N., "Prevalence of haptic feedback in robot-mediated surgery: a systematic review of literature," Journal of Robotic Surgery, 12, (2018) Isue 1, 11-25 